\title{
Fe-Ti-O based catalyst for large-chiral-angle single-walled carbon nanotube growth
}

He, Maoshuai; Zhang, Lili; Jiang, Hua; Yang, He; Fossard, Frédéric; Cui, Hongzhi; Sun, Zhipei; Wagner, Jakob Birkedal; Kauppinen, Esko I.; Loiseau, Annick

\section{Published in:}

Carbon

Link to article, DOI:

10.1016/j.carbon.2016.06.099

Publication date:

2016

Document Version

Peer reviewed version

Link back to DTU Orbit

Citation (APA):

He, M., Zhang, L., Jiang, H., Yang, H., Fossard, F., Cui, H., Sun, Z., Wagner, J. B., Kauppinen, E. I., \& Loiseau, A. (2016). Fe-Ti-O based catalyst for large-chiral-angle single-walled carbon nanotube growth. Carbon, 107, 865-871. https://doi.org/10.1016/j.carbon.2016.06.099

\section{General rights}

Copyright and moral rights for the publications made accessible in the public portal are retained by the authors and/or other copyright owners and it is a condition of accessing publications that users recognise and abide by the legal requirements associated with these rights.

- Users may download and print one copy of any publication from the public portal for the purpose of private study or research.

- You may not further distribute the material or use it for any profit-making activity or commercial gain

- You may freely distribute the URL identifying the publication in the public portal 


\section{Fe-Ti-O Based Catalyst for Large-Chiral-Angle Single-Walled Carbon Nanotube Growth}

Maoshuai He, ${ }^{a, b^{*}}$ Lili Zhang, ${ }^{c}$ Hua Jiang, ${ }^{d}$ He Yang, ${ }^{e}$ Frédéric Fossard, ${ }^{a}$ Hongzhi Cui, ${ }^{*}$ Zhipei Sun, ${ }^{e}$ Jakob B. Wagner, ${ }^{c}$ Esko I. Kauppinen, ${ }^{e}$ Annick Loiseau ${ }^{b}$

a School of Materials Science and Engineering, Shandong University of Science and Technology, 266590 Qingdao, People’s Republic of China

b Laboratoire d'Étude des Microstructures, ONERA-CNRS, BP 72, 92322 Châtillon CEDEX, France

c Center for Electron Nanoscopy, Technical University of Denmark, DK-2800 Kongens Lyngby, Denmark

${ }^{\mathrm{d}}$ Department of Applied Physics, Aalto University School of Science, P.O. Box 15100, FI-00076 Aalto, Finland

e Department of Micro and Nanosciences, Aalto University, P.O. Box 13500, FI-00076, Aalto, Finland

\section{Corresponding Author}

* Correspondence should be addressed to: Maoshuai He, Email: maoshuai.he@sdust.edu.cn, Tel:+86 53280691718; Hongzhi Cui, Email: cuihongzhi1965@163.com, Tel:+86 53280681145 


\section{Abstract:}

Catalyst selection is very crucial for controlled growth of single-walled carbon nanotubes (SWNTs). Here we introduce a well-designed Fe-Ti-O solid solution for SWNT growth with a high preference to large chiral angles. The Fe-Ti-O catalyst was prepared by combining Ti layer deposition onto premade Fe nanoparticles with subsequent high-temperature air calcination, which favours the formation of a homogeneous Fe-Ti-O solid solution. Using $\mathrm{CO}$ as the carbon feedstock, chemical vapour deposition growth of SWNTs at $800{ }^{\circ} \mathrm{C}$ was demonstrated on the Fe-Ti-O catalyst. Nanobeam electron diffraction characterization on a number of individual SWNTs revealed that more than $94 \%$ of SWNTs have chiral angles larger than $15^{\circ}$. In situ environmental transmission electron microscopy study was carried out to reveal the catalyst dynamics upon reduction. Our results identify that the phase segregation through reducing Fe-Ti-O catalyst leads to the formation of $\mathrm{TiO}_{\mathrm{x}}$-supported small Fe nanoparticles for SWNT growth. The strong metal-support interactions induced by partial reduction of $\mathrm{TiO}_{\mathrm{x}}$ support promote the wettability of Fe nanoparticle, accounting for the preferential growth of large-chiral-angle SWNTs. This work opens a new avenue for chiral angle selective growth of SWNTs.

Keywords: single-walled carbon nanotube, Fe-Ti-O catalyst, environmental transmission electron microscope, chemical vapor deposition, large chiral angle

\section{Introduction:}

With the aim to exploit potential technological applications of single-walled carbon nanotubes (SWNTs), it is necessary to synthesize SWNTs with well-defined structures $[1,2]$. This is due to the fact that the unique fundamental properties of an SWNT, like electrical and optical properties, derive from the fashion of folding carbon sheet described by the tube chirality. A pair of chiral indices $(n, m)$ is commonly used to define the tube chirality. Highly crystalline SWNTs can be produced by high temperature techniques, such as arc discharge [3,4] and laser ablation [5] methods. However, these 
methods usually yield SWNTs with mixed properties. Moreover, SWNTs produced by these high temperature techniques typically need to be dispersed onto desirable substrates for further device fabrication. Nowadays, chemical vapour deposition (CVD) technique has become the most widespread method for directly synthesizing SWNTs on substrate/support, since the orientation [6-8], density [9, 10], diameter [11] and even chirality $[12,13]$ can all be controlled to a certain extent.

As a subgoal of fully control the final structure of SWNTs, controlling the chiral angles of SWNTs has been challenging [14-17]. Indeed, many growth parameters involved in CVD, including catalyst composition [12, 13, 18-20], catalyst state [17], carbon source [21, 22], reaction temperature [12, 13, $23,24]$ and metal-support interactions [25, 26], all affect the SWNT growth results, making chiralselective SWNT growth extremely difficult. Among all the above parameters, catalyst is known as the most important parameter in defining SWNT chirality distribution. Since the pioneer work of CoMoCAT catalyst [18], a number of catalyst systems have been proposed for chiral-selective SWNT growth in the past decade. However, in most instances, the chirality selectivity has been limited to low temperature growth of small diameter SWNTs [12, 18, 24]. Therefore, it is still highly desired to develop catalyst systems suitable for growing SWNTs with relatively large diameters and certain chiral-angle bias.

Solid solutions arise recently as promising catalysts for achieving SWNTs with specific chiralities $[13,19]$. This is because the solid solution offers additional degree of freedom for regulating the morphology and structure of the active catalyst component. For example, by tuning the synthesis temperatures, SWNTs enriched with different tube types were obtained on a $\mathrm{Co}_{\mathrm{x}} \mathrm{Mg}_{1-\mathrm{x}} \mathrm{O}$ solid solution [19]. Reduction of such a $\mathrm{Co}_{\mathrm{x}} \mathrm{Mg}_{1-\mathrm{x}} \mathrm{O}$ catalyst leads to the epitaxial formation of uniform Co nanoparticles and the concomitant strong metal-support interactions (SMSI), accounting for the high SWNT selectivity to near-armchair species. SMSI is thus anticipated to favor the synthesis of SWNTs with bias chirality. It has been long recognized that $\mathrm{TiO}_{2}$ supported metals (like Fe) possess SMSI [27] 
and show impressive properties in Fischer-Tropsch [28, 29] and carbon nanotube growth [30, 31]. However, on the one hand, the structures and dynamics of Fe-Ti-O catalyst upon reduction are still unveiled; on the other hand, the development of $\mathrm{TiO}_{2}$-based solid solution for the synthesis of SWNTs, especially SWNTs with certain chiral angle selectivity, has been scarce.

In this work, we present a Fe-Ti-O solid solution catalyst for synthesizing SWNTs with a high preference to large chiral angles. The Fe-Ti-O solid solution is obtained by high-temperature calcination of Ti-covered Fe nanoparticles, which are supported by porous $\mathrm{Si}_{3} \mathrm{~N}_{4}$ grid. The chiralities of SWNTs crossing the grid holes will be identified by nanobeam electron diffraction (ED) and compared with those grown on monometallic Fe catalyst. In order to understand the SWNT growth mechanism, the reduction behavior of the Fe-Ti-O catalyst will be elucidated with the assistance of in situ environmental transmission electron microscopy (E-TEM). The SMSI is postulated to play an important role in the chiral-angle selective growth of SWNTs.

\section{Materials and methods}

\subsection{Preparation and characterization of Fe-Ti-O catalyst}

Fig. 1 depicts the preparation of the Fe-Ti-O solid solution. The $\mathrm{FeO}_{\mathrm{x}}$ nanoparticles were prepared by hydrolysis of anhydrous ferric chloride $\left(\mathrm{FeCl}_{3}\right)$ [32]. Briefly, $\mathrm{FeCl}_{3}$ with a mass of $0.807 \mathrm{~g}$ was dissolved in $5 \mathrm{~mL} \mathrm{H}_{2} \mathrm{O}$ to form a clear solution. The aqueous solution of $\mathrm{FeCl}_{3}$ was added dropwise into $180 \mathrm{~mL}$ boiling $\mathrm{H}_{2} \mathrm{O}$ under vigorous stirring and refluxed for $2 \mathrm{~h}$ to form $\mathrm{FeO}_{\mathrm{x}}$ nanoparticles. Asprepared $\mathrm{FeO}_{x}$ nanoparticles were casted onto a $\mathrm{Si}_{3} \mathrm{~N}_{4}$ TEM grid (DuraSiN mesh). After that, the grid was deposited with $4 \mathrm{~nm}$ Ti (Edwards E306A Evaporator e-gun system). The system pressure was kept at $10^{-6} \mathrm{~Pa}$ during Ti deposition. The catalyst was finally annealed at $900{ }^{\circ} \mathrm{C}$ for $20 \mathrm{~h}$ in air to facilitate the formation of Fe-Ti-O solid solution. 
The morphology and structure of the Fe-Ti-O catalyst were characterized by a Zeiss LIBRA 200FMC TEM operated with an accelerating voltage of $200 \mathrm{kV}$. Electron energy-loss spectroscopy (EELS) was applied to analyse the elemental components of the Fe-Ti-O particles.

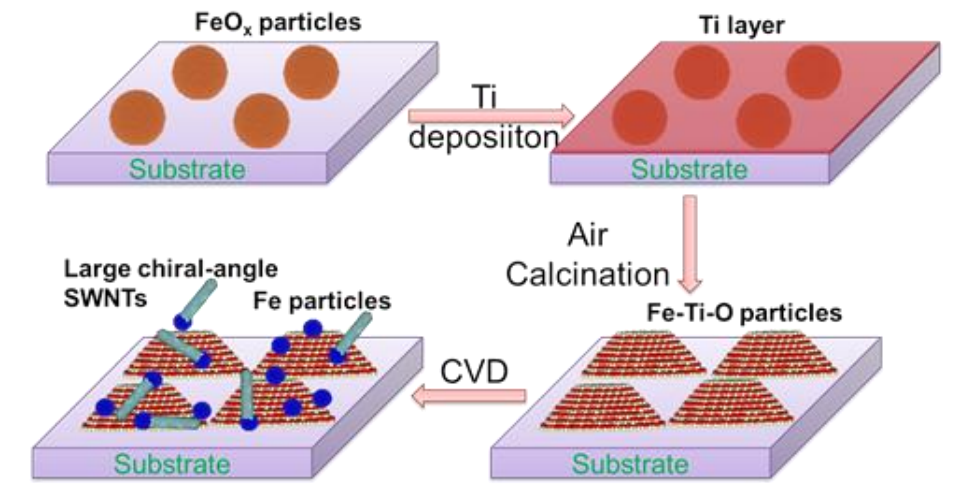

Fig. 1 Schematic illustrations of preparing Fe-Ti-O solid solution catalyst for CVD growth of SWNTs.

\subsection{Growth and characterization of carbon nanotubes}

The $\mathrm{Si}_{3} \mathrm{~N}_{4}$ supported Fe-Ti-O catalyst was loaded into a horizontal CVD reactor with a quartz tube (inner diameter $40 \mathrm{~mm}$ ). The system was heated to $800{ }^{\circ} \mathrm{C}$ in the protection of helium with a flow rate of $200 \mathrm{~cm}^{3} / \mathrm{min}$. After being stabilized at $800{ }^{\circ} \mathrm{C}$, helium was replaced by $\mathrm{CO}\left(200 \mathrm{~cm}^{3} / \mathrm{min}\right)$ for SWNT growth. The feeding of $\mathrm{CO}$ at $800{ }^{\circ} \mathrm{C}$ lasted for $1 \mathrm{~h}$. The reactor was cooled to room temperature in flowing helium.

As-prepared carbon nanotubes were characterized with Raman spectrometer with an excitation laser wavelength of $632.8 \mathrm{~nm}$ (Horiba Jobin-Yvon LabRam HR800) and scanning electron microscopy (SEM, JEOL JSM-7500F). A JEOL-2200FS double aberration-corrected TEM operated at $80 \mathrm{kV}$ was applied to investigate the carbon nanotubes. The chiralities of SWNTs were identified from their ED patterns on the basis of a calibration-free intrinsic layer line-spacing method [33].

\subsection{In situ E-TEM investigations on the catalyst dynamics upon reduction}

In situ experiment was performed on an FEI Titan 80-300ST E-TEM operated at an accelerating voltage of $300 \mathrm{kV}$. The Fe-Ti-O catalyst supported by $\mathrm{Si}_{3} \mathrm{~N}_{4}$ grid was loaded onto an Inconel heating 
holder and inserted into the TEM chamber. Once heated up to $700{ }^{\circ} \mathrm{C}$, $\mathrm{CO}$ with a flow rate of 6 $\mathrm{cm}^{3} / \mathrm{min}$ was introduced to the chamber and the pressure was stabilized at $7.0 \mathrm{mbar}$. The catalyst dynamics upon $\mathrm{CO}$ reduction was monitored by TEM imaging.

\section{Results and Discussion}

\subsection{Preparation and characterization of Fe-Ti-O solid solution}

Supporting Information Fig. S1a presents a TEM overview of $\mathrm{FeO}_{\mathrm{x}}$ nanoparticles prepared by hydrolysis of $\mathrm{FeCl}_{3}$. After their dispersion onto the surface of $\mathrm{Si}_{3} \mathrm{~N}_{4} \mathrm{TEM}$ grid, a layer of $4 \mathrm{~nm}$ Ti was deposited onto the entire surface. When the $\mathrm{Ti}$ coated $\mathrm{FeO}_{\mathrm{x}}$ nanoparticles were heated in air at $900{ }^{\circ} \mathrm{C}$, the mobility of $\mathrm{Ti}$ atoms increases, leading to the formation of $\mathrm{Fe}-\mathrm{Ti}-\mathrm{O}$ particles due to surface immigration [34]. A TEM image of the catalyst after air calcination is presented in Supporting Information Fig. S1b. Clearly, most of the particles after air calcination have sizes of several tens of nm. Fig. 2a presents a TEM image of an ellipse-shaped catalyst particle, showing a perfect single crystalline structure. The major and minor axes of the particle are $28 \mathrm{~nm}$ and $19 \mathrm{~nm}$, respectively. EELS (Fig. 2b) acquired on as-prepared catalyst particle distinctively show the $\mathrm{Ti} \mathrm{L}_{2}$ and $\mathrm{L}_{3}$ major edges at $455 \mathrm{eV}$ and $461 \mathrm{eV}$, respectively. Besides, the O_K edge at $532 \mathrm{eV}$, the $\mathrm{L}_{2}(702 \mathrm{eV})$ and $\mathrm{L}_{3}$ $(715 \mathrm{eV})$ edges of Fe are also present.

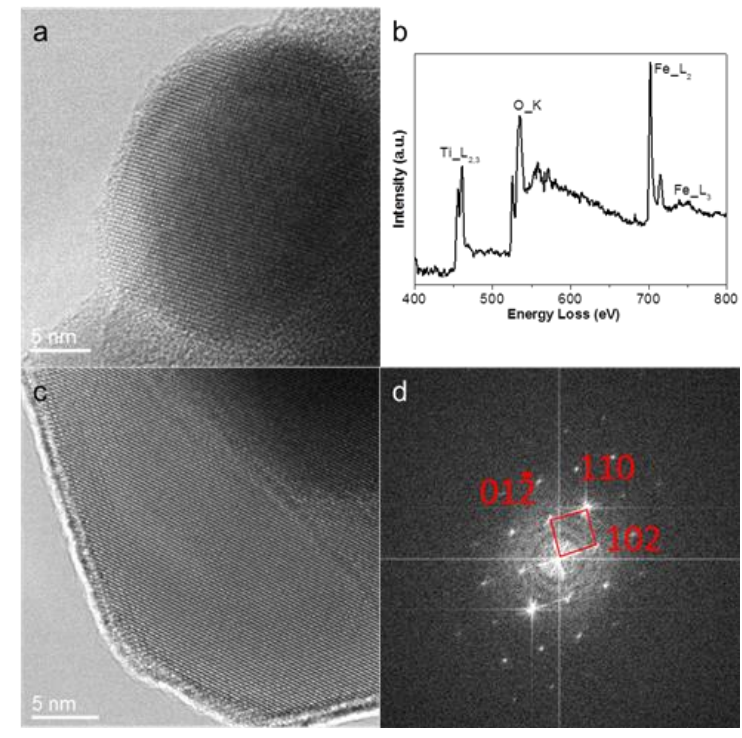


Fig. 2 (a) TEM image of a typical Fe-Ti-O catalyst particle. (b) Electron energy loss spectrum taken from a Fe-Ti-O particle. (c) TEM image of another Fe-Ti-O particle and (d) its corresponding fast Fourier transformation digital diffractogram.

Fig. 2c and Fig. 2d present high resolution TEM image of a large Fe-Ti-O particle and its corresponding fast Fourier transformation (FFT) pattern, respectively. Our high resolution TEM image fully confirms a single crystalline structure of the Fe-Ti-O catalyst. Combined with its FFT pattern, the crystal structure was assigned as $\mathrm{FeTiO}_{3}$ with a trigonal rhombohedral structure $(\mathrm{a}=\mathrm{b}=0.508 \mathrm{~nm}, \mathrm{c}=$ $1.42 \mathrm{~nm}$ ). The respective interplanar spacing of (102) and (110) are $0.37 \mathrm{~nm}$ and $0.25 \mathrm{~nm}$ (Fig. 2d). All the above results suggest that particles of Fe-Ti-O solid solution are formed on the surface of $\mathrm{Si}_{3} \mathrm{~N}_{4}$ grid. 3.2. Synthesis and characterizations of SWNTs grown on Fe-Ti-O catalyst

Ambient pressure CVD growth of carbon nanotube was performed at $800{ }^{\circ} \mathrm{C}$ using $\mathrm{CO}$ as the carbon source. Supporting Information Fig. S2a presents radial breath modes of Raman spectra acquired at $632.8 \mathrm{~nm}$ on the carbon nanotube products, indicating the synthesis of SWNTs. The relatively low density of D mode (Supporting Information Fig. S2b) suggests that the quality of produced SWNTs is high. It is noted that Raman scattering is a resonant process. That is, when using a fixed laser wavelength, only a small portion of SWNTs with certain bandgaps can be detected by Raman spectroscopy [35]. In addition, the Raman frequencies of SWNTs are sensitive to environments, challenging the precise assignment of the chiral indices of SWNTs.

Fig. 3a and Supporting Information Fig. S3a presents a TEM overview and a SEM overview of as-prepared carbon nanotubes grown on Fe-Ti-O catalyst, respectively. High-resolution TEM (Supporting Information Fig. S3b) and ED characterization results confirm that the synthesized nanotubes are single-walled. Fig. 3b shows the diffraction pattern of an individual SWNT (inset of Fig. 3a). The diffraction layer lines are separated by certain distances from the equatorial layer line at the centre. The period of the equatorial line is related to the diameter of the tube $[33,36]$. Coupled with 
the spacing of non-equatorial lines, the chirality of an SWNT can be well assigned. For example, Fig. 3b gives the SWNT chirality of $(10,8)$, indicating that the SWNT is a semiconducting tube with a chiral angle of $26.3^{\circ}$. Additional confirmation of the assignment is provided by the simulated ED pattern of a $(10,8)$ tube (right of Fig. 3b).
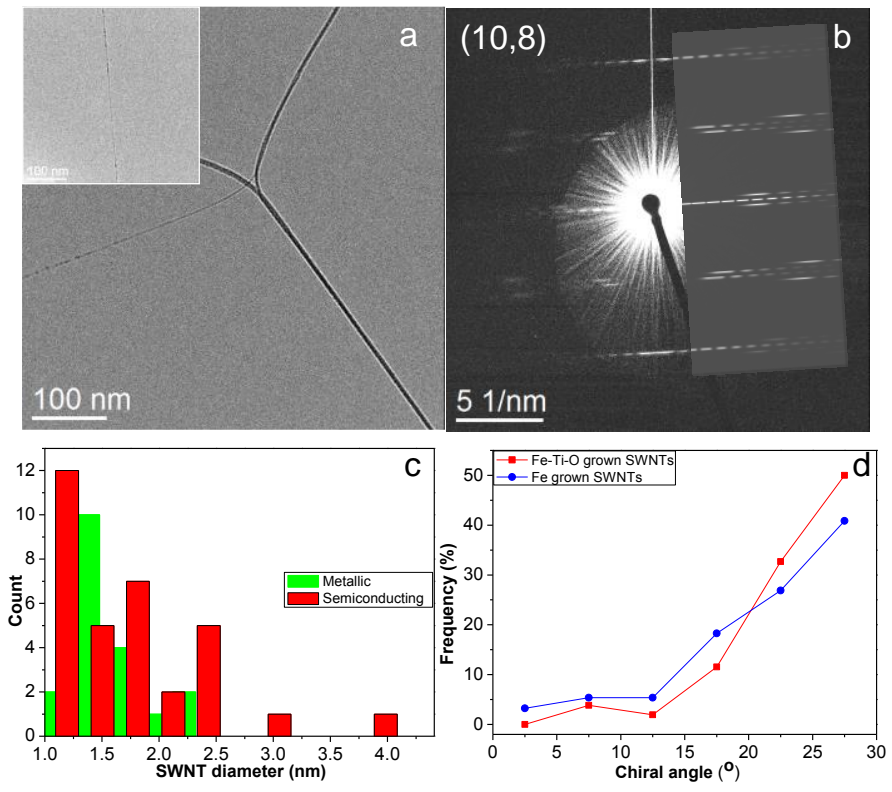

Fig. 3 (a) A TEM overview of as synthesized carbon nanotubes grown on Fe-Ti-O catalyst at $800{ }^{\circ} \mathrm{C}$ using $\mathrm{CO}$ as the carbon source. (b) The ED pattern of an individual SWNT (inset of Figure (a)). The right is the simulated diffraction pattern of a $(10,8)$ tube. (c) Diameter distribution of metallic and semiconducting SWNTs grown on Fe-Ti-O. (d) The distributions of SWNTs grown from Fe-Ti-O and Fe as a function of chiral angles.

Nanobeam ED characterization was performed on a total of 52 randomly isolated SWNTs. The deduced $(n, m)$ indices, chiral angles and diameters of SWNTs are all listed in Supporting Information Table S1. There is no preference to tubes with metallic or semiconducting indices. 19 out of 52 SWNTs were identified to be metallic species, comprising $36 \%$ of the total, which is close to the theoretically predicted amount (33\%). Supporting Information Fig. S4a presents the diameter 
distribution of SWNTs. Most of SWNTs (96\%) have diameters in the range of $1.0-2.4 \mathrm{~nm}$, as also demonstrated in Fig. 3c. Although no high chiral selectivity is observed, $(14,8)$ tube with a chiral angle of $21.1^{\circ}$, is the most abundant species detected in the product. Besides, SWNTs with diameters near 1.2 and $1.3 \mathrm{~nm}$, including $(10,8),(10,9),(11,7)$ and $(11,8)$ were also observed more frequently than other tube species, occupying $23 \%$ of all the SWNTs.

Furthermore, Fig. 3c depicts that there is no simple correlation between the tube diameter and tube conductivity. Interestingly, most of the tubes (up to 94\%) have chiral angle larger than $15^{\circ}$ and the mean angle is $24^{\circ}$ with a standard deviation of $5^{\circ}$. As suggested by Supporting Information Fig. S4b and Table S1, only 3 SWNTs, namely one $(15,2)$, one $(23,7)$ and one $(33,7)$ tube, have chiral angles smaller than $15^{\circ}$. Fig. 3d compares the percentages of Fe-Ti-O grown SWNTs in different chiral angle ranges with those of SWNTs grown on monometallic Fe catalyst [22]. Clearly, SWNTs with large chiral angles $\left(20^{\circ}-30^{\circ}\right)$ were more frequently detected in the Fe-Ti-O grown products $(82 \%)$.

To help understanding of the above SWNT growth results, nucleation and growth mechanisms of SWNTs should be considered. The growth of an SWNT starts with the nucleation of an SWNT cap, while the position of the sixth pentagon in the cap determines the chiral angle of an SWNT. A few parameters, including gas environments, catalyst composition and metal-support interaction, all could influence the formation of tube cap. In our CVD experiment, pure $\mathrm{CO}$, which has been demonstrated to favour the selective nucleation of certain SWNT caps [22, 37], was applied as the carbon precursor. However, as shown in Fig. 3d, the portion of large-chiral-angle SWNTs grown on Fe-Ti-O is higher than that of monometallic Fe-grown SWNTs [22]. Therefore, the use of CO is not fully responsible for the preferential growth of large-chiral-angle SWNTs on Fe-Ti-O catalyst.

\subsection{In situ E-TEM studies on the behaviours of Fe-Ti-O catalyst upon reduction}

Alloying with high melting temperature metal/metal oxide could affect the catalytic performances of Fe-group transition metal (Fe, Co and Ni). For example, CVD growth of SWNTs with high chirality 
selectivity was reported on solid solutions like $\mathrm{Co}_{\mathrm{x}} \mathrm{Mg}_{1-\mathrm{x}} \mathrm{O}[19,26]$ and $\mathrm{Co}-\mathrm{W}$ [13]. However, the catalytic performances of the above two catalysts are quite different. When reducing the $\mathrm{Co}-\mathrm{MgO}$ catalyst, Co cations in the solid phase are reduced and migrated into the surface to form metallic Co nanoparticles [19] which catalyse the subsequent growth of SWNTs. While Co-W catalyst nanoparticles appeared to be very stable and no phase separation occurs when heated up to $1100{ }^{\circ} \mathrm{C}[13]$.

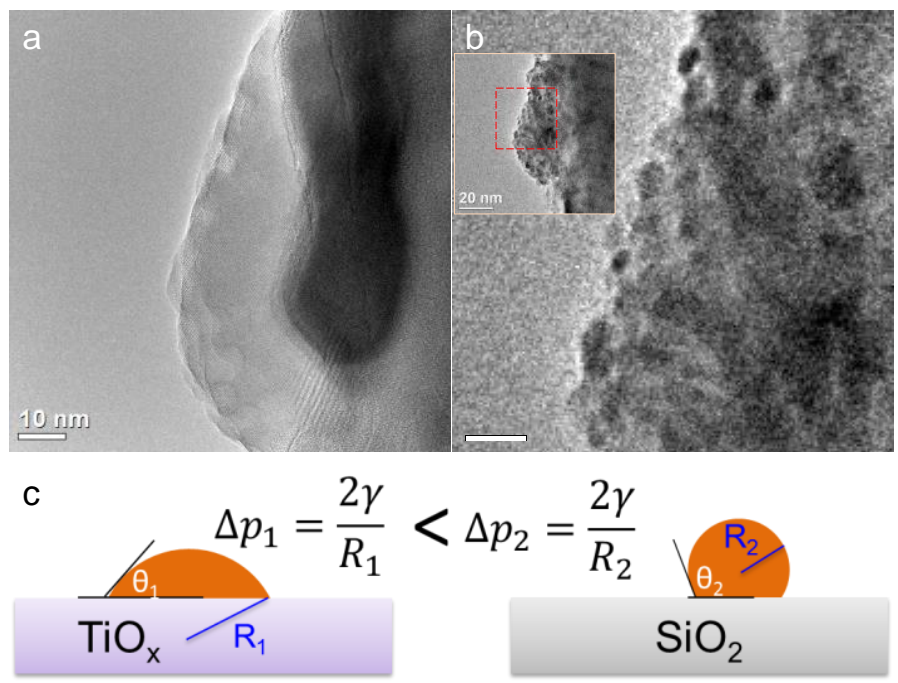

Fig. 4 (a) TEM image of a Fe-Ti-O particle. (c) TEM images of the same particle shown in (a) after in situ reduction in $\mathrm{CO}$ at $700{ }^{\circ} \mathrm{C}$. Inset is the overview of the particle. (c) Schematic illustration of the enhanced wettability of $\mathrm{Fe}$ nanoparticles on $\mathrm{TiO}_{\mathrm{x}}$ support, resulting in less Laplace pressure of $\mathrm{Fe}$ nanoparticles.

In order to figure out what is the active composition for growing SWNTs and address why Fe-Ti-O particles can lead to the growth of SWNTs with large chiral angles, in situ E-TEM experiments were performed to investigate the reductive behaviours of the Fe-Ti-O catalyst. Fig. 4a presents a TEM image of a representative Fe-Ti-O particles before reduction. No small particles anchor on the surfaces of Fe-Ti-O solid solution. After in situ reduction by $\mathrm{CO}$ at $700{ }^{\circ} \mathrm{C}$, Fe particles with small diameters were observed to form on the surfaces of Fe-Ti-O particles (Fig. 4b). Such observations are in 
agreement with the work reported by Campos et al. [38], who detected the formation of metallic Fe on reduced Fe-Ti-O solid solution using XRD. All the above results suggest that the catalyst behaviours of Fe-Ti-O are similar to those of $\mathrm{Co}_{\mathrm{x}} \mathrm{Mg}_{1-\mathrm{x}} \mathrm{O}$ type solid solution, where phase separation occurs upon reduction at high temperature.

As the active component of the Fe-Ti-O solid solution for nucleating SWNTs is Fe nanoparticles, it seems reasonable to understand that SWNTs grown on Fe-Ti-O display no metallic property tuning compared with Fe-grown SWNTs [22]. Further considering both the carbon precursor and the reaction temperature are same, we tentatively attribute the pronounced growth of large-chiral-angle SWNTs on Fe-Ti-O to the effects of the support materials. On the one hand, the acid-base character of the support surface could influence the carbon source chemistry. $\mathrm{TiO}_{2}$ supported Fe catalyst has been explored in Fischer-Tropsch synthesis where $\mathrm{CO}$ is used as one of the starting materials. Analyzed by a gas chromatograph, Arai et al.[28] discovered that $\mathrm{Fe} / \mathrm{TiO}_{2}$ catalyst showed a high selectivity to $\mathrm{C}_{1}-\mathrm{C}_{5}$ hydrocarbons and no $\mathrm{C}_{6+}$ hydrocarbons were produced. Compared with Fischer-Tropsch synthesis [28], the temperature $\left(800{ }^{\circ} \mathrm{C}\right)$ used in our CVD process is higher. Such a high temperature not only facilitates faster reaction and higher conversion rates but favours the production of $\mathrm{C}_{1}$ hydrocarbon. Such short carbon chains could act as intermediates and be directly attached to the open ends of SWNTs. More importantly, the carbon fragments generated when using $\mathrm{TiO}_{2}$ as the support could affect the carbon dissolution in the $\mathrm{Fe}$ nanoparticles and therefore, the subsequent nucleation of a carbon nanotube.

On the other hand, metal-support interaction could regulate the morphology of supported particles [39], which influences the formation of a SWNT cap. By controlling the rearrangement of catalyst particles, growth of SWNTs with different structures was reported by Harutyunyan et al.[40] They demonstrated that there exist correlations between catalyst morphology and the resulted nanotube electronic structure. Our recent work also shows that the carbon incorporation depends on the 
nanoparticle structures [41], thus affecting the nucleation of SWNTs. In the case of Fe-Ti-O catalyst, as evidenced by E-TEM experiments, reduced Fe anions migrate onto the surface to form metallic Fe nanoparticles. Different from oxides such as $\mathrm{SiO}_{2}, \mathrm{Al}_{2} \mathrm{O}_{3}$ and $\mathrm{MgO}$, which usually show weak metalsupport interactions, $\mathrm{TiO}_{2}$ support could exhibits SMSI with reduced Fe nanoparticles [27]. The occurrence of SMSI correlates with the partial reduction of surface $\mathrm{TiO}_{2}$ to " $\mathrm{TiO}_{\mathrm{x}}$ " which converts $\mathrm{Ti}^{4+}$ with a $\mathrm{d}^{0}$ configuration to $\mathrm{Ti}^{3+}$ with a $\mathrm{d}^{1}$ configuration [42]. In the support surface, a local anion deficiency allows the reduced $\mathrm{Fe}$ atoms migrate to freshly reduced region of the adjacent $\mathrm{TiO}_{\mathrm{x}}$. The strong strength of the interaction between the metal and the support not only inhibits agglomeration of the metal particles under reaction condition, but induces the metallic particle to adopt a pillbox-like morphology (Fig. 4b) that maximizes the number of metal atoms in contact with the support [43]. The SMSI-induced pillbox-like morphology should increase the proportion of low-index planes [42] and eventually result in the growth of SWNTs different from those grown on $\mathrm{Si}_{3} \mathrm{~N}_{4}$ or $\mathrm{SiO}_{2}$ supported Fe nanoparticles. In addition, the SMSI promotes the wettability of Fe nanoparticles on the substrate and leads to the reduced contacted angle of the nanoparticles. The curvature of Fe nanoparticles decreases accordingly, which results in less Laplace pressure of liquid Fe nanoparticles (Fig. 4c) and thus limits their entrance into the carbon nanotubes. As a result, the tube cap structure formed on the Fe nanoparticle differs from that produced on $\mathrm{SiO}_{2}$ supported $\mathrm{Fe}$, where exists a large contact angle between Fe and substrate [44]. Such an effect could also be induced by modulating carbon content inside metal particles, which has been demonstrated to be of great importance for carbon nanotube nucleation and growth $[41,45]$. Indeed, previous work also revealed that metallic Co nanoparticles, which have strong interactions with the underlying $\mathrm{MgO}$ support and favor the growth of near-armchair SWNTs $[19,26]$.

\section{Conclusions}


In conclusion, a facile deposition and calcination technique was developed to prepare a $\mathrm{Fe}-\mathrm{Ti}-\mathrm{O}$ solid solution for catalysing the growth of SWNTs. ED characterizations on the SWNTs revealed a preferential growth of SWNTs with large chiral angles. In situ E-TEM investigations revealed that the active catalyst for growing SWNTs is Fe nanoparticles, generated from migration of $\mathrm{Fe}$ ions in the solid solution upon high temperature reduction. The presence of SMSI induced by $\mathrm{TiO}_{\mathrm{x}}$ support lead to the formation of metallic Fe nanoparticles with unique morphologies and enhanced wettability of $\mathrm{Fe}$ nanoparticle on support, accounting for such SWNT growth results. This work extends the development of solid solution catalysts for CVD synthesis of SWNTs and sheds more light on chiralselective growth of SWNTs.

\section{Acknowledgements}

The research leading to these results has received funding from the European Union Seventh Framework Programme (FP7/2007-2013) under grant agreement $\mathrm{n}^{\circ} 604472$ (IRENA project), by the Aalto Energy Efficiency (AEF) program through the MOPPI project, from TEKES via CARLA and USG projects and from Academy of Finland via HISCON project. The authors would also like to acknowledge Scientific Research Foundation of Shandong University of Science and Technology for Recruited Talents, the Natural Science Foundation of China (No. 51272141) and Taishan Scholars Project of Shandong (No. TS20110828). He Yang acknowledges funding from China Scholarship Council. Dr. Xuelin Tian is acknowledged for helpful discussion. The work made use of the Aalto University Nanomicroscopy Center (Aalto-NMC) and Micronova Nanofabridcation Center premises.

Supplementary data: Extensive figures and results related to catalyst and carbon nanotube characterizations.

\section{References}


[1] Baughman R H, Zakhidov A A and de Heer W A, Carbon nanotubes--the route toward applications, Science, 2002; 297: 787-92.

[2] Tans S J, Verschueren A R M and Dekker C, Room-temperature transistor based on a single carbon nanotube Nature, 1998; 393: 49-52.

[3] Iijima S and Ichihashi T, Single-shell carbon nanotubes of 1-nm diameter, Nature, 1993; 363: 603-5.

[4] Bethune D S, Kiang C H, de Vries M S, Gorman G, Savoy R, Vazquez Z, et al., cobalt-catalysed growth of carbon nanotubes with single-atomic-layer walls, Nature, 1993; 363: 605-7.

[5] Guo T, Nikolaev P, Thess A, Colbert D and Smalley R, Catalytic growth of single-walled manotubes by laser vaporization, Chem Phys Lett, 1995; 243: 49-54.

[6] Zhang Y, Chang A, Cao J, Wang Q, Kim W, Li Y, et al., Electric-field-directed growth of aligned single-walled carbon nanotubes, Appl Phys Lett, 2001; 79: 3155-7.

[7] Huang S, Cai X and Liu J, Growth of millimeter-long and horizontally aligned single-walled carbon nanotubes on flat substrates, J Am Chem Soc, 2003; 125: 5636-7.

[8] Ismach A, Segev L, Wachtel E and Joselevich E, Atomic-step-templated formation of single wall carbon nanotube patterns, Angew Chem Int Ed, 2004; 116: 6266-9.

[9] Ding L, Yuan D and Liu J, Growth of high-density parallel arrays of long single-walled carbon nanotubes on quartz substrates, J Am Chem Soc, 2008; 130: 5428-9.

[10] Hu Y, Kang L, Zhao Q, Zhong H, Zhang S, Yang L, et al., Growth of high-density horizontally aligned SWNT arrays using Trojan catalysts, Nature Commun, 2015; 6: 6099.

[11] Cheung C L, Kurtz A, Park H and Lieber C M, Diameter-controlled synthesis of carbon nanotubes, J Phys Chem B, 2002; 106: 2429-33.

[12] He M, Chernov A I, Fedotov P V, Obraztsova E D, Sainio J, Rikkinen E, et al., Predominant $(6,5)$ single-walled carbon nanotube growth on a copper-promoted iron catalyst, J Am Chem Soc, 2010; 132: 13994-6. 
[13] Yang F, Wang X, Zhang D, Yang J, Luo D, Xu Z, et al., Chirality-specific growth of single-walled carbon nanotubes on solid alloy catalysts, Nature, 2014; 510: 522-4.

[14] Paillet M, Meyer J C, Michel T, Jourdain V, Poncharal P, Sauvajol J-L, et al., Selective growth of large chiral angle single-walled carbon nanotubes, Diamond Relat Mater, 2006; 15: 1019-22.

[15] He M, Jiang H, Lehtonen J and Kauppinen E I, Growth of single-walled carbon nanotubes with large chiral angles on rhodium nanoparticles, Nanoscale, 2013; 5: 10200-2.

[16] Zhu Z, Jiang H, Susi T, Nasibulin A G and Kauppinen E I, The Use of NH3 to promote the production of large-diameter single-walled carbon nanotubes with a narrow $(\mathrm{n}, \mathrm{m})$ distribution, $\mathrm{J}$ Am Chem Soc, 2011; 133: 1224-7.

[17] Alemán B, Bernal M M, Mas B, Perez E M, Reguero V, Xu G, et al., Inherent predominance of high chiral angle metallic carbon nanotubes in continuous fibers grown from molten catalyst, Nanoscale, 2016; 8: 4236-44.

[18] Bachilo S M, Balzano L, Herrera J E, Pompeo F, Resasco D E and Weisman R B, Narrow (n,m)distribution of single-walled carbon nanotubes grown using a solid supported catalyst, J Am Chem Soc, 2003; 125: 11186-7.

[19] He M, Jiang H, Liu B, Fedotov P V, Chernov A I, Obraztsova E D, et al., Chiral-selective growth of single-walled carbon nanotubes on lattice-mismatched epitaxial cobalt nanoparticles, Sci Rep, 2013; 3: 1460.

[20] Orbaek A W, Owens A C, Crouse C C, Pint C L, Hauge R H and Barron A R, Single walled carbon nanotube growth and chirality dependence on catalyst composition, Nanoscale, 2013; 5: 984859.

[21] Wang B, Poa C H P, Wei L, Li L-J, Yang Y and Chen Y, (n,m) Selectivity of single-walled carbon nanotubes by different carbon precursors on Co-Mo catalysts, J Am Chem Soc, 2007; 129: 9014-9. 
[22] He M, Jiang H, Kauppinen E I and Lehtonen J, Diameter and chiral angle distribution dependencies on the carbon precursors in surface-grown single-walled carbon nanotubes, Nanoscale, 2012; 4: 7394-8.

[23] He M, Chernov A I, Obraztsova E D, Sainio J, Rikkinen E, Jiang H, et al., Low temperature growth of SWNTs on a nickel catalyst by thermal chemical vapor deposition, Nano Res, 2011; 4: 33442.

[24] Li X, Tu X, Zaric S, Welsher K, Seo W S, Zhao W, et al., Selective synthesis combined with chemical separation of single-walled carbon nanotubes for chirality selection, J Am Chem Soc, 2007; 129: $15770-1$.

[25] Ago H, Nakamura K, Uehara N and Tsuji M, Roles of metal-support interaction in growth of single-and double-walled carbon nanotubes studied with diameter-controlled iron particles supported on MgO, J Phys Chem B, 2004; 108: 18908-15.

[26] He M, Jiang H, Kauppi I, Fedotov P V, Chernov A I, Obraztsova E D, et al., Insights into chirality distributions of single-walled carbon nanotubes grown on different $\mathrm{CoxMg1-xO}$ solid solutions, $\mathrm{J}$ Mater Chem A, 2014; 2: 5883-9.

[27] Rao P M, Viswanathan B and Viswanath R, Strong metal support interaction state in the Fe/TiO2 system—an XPS study, J Mater Sci, 1995; 30: 4980-5.

[28] Arai H, Mitsuishi K and Seiyama T, TiO2-supported Fe-Co,Co-Ni, and Ni-Fe alloy catalysts for Fischer-Tropsch synthesis, Chem Lett, 1984; 13: 1291-4.

[29] Duvenhage D and Coville N, Fe: Co/TiO 2 bimetallic catalysts for the Fischer-Tropsch reaction: part 3: the effect of Fe: Co ratio, mixing and loading on FT product selectivity, Appl Catal A Gen, 2005; 289: $231-9$. 
[30] Gunjishima I, Inoue T, Yamamuro S, Sumiyama K and Okamoto A, Growth of vertically aligned carbon nanotubes from highly active Fe-Ti-O nanoparticles prepared by liquid-phase synthesis, Jpn $\mathbf{J}$ Appl Phys, 2007; 46: 3700-3.

[31] Okamoto A, Gunjishima I, Inoue T, Akoshima M, Miyagawa H, Nakano T, et al., Thermal and electrical conduction properties of vertically aligned carbon nanotubes produced by water-assisted chemical vapor deposition, Carbon, 2011; 49: 294-8.

[32] He M, Duan X, Wang X, Zhang J, Liu Z and Robinson C, Iron catalysts reactivation for efficient CVD growth of SWNT with base-growth mode on surface, J Phys Chem B, 2004; 108: 12665-8.

[33] Jiang H, Nasibulin A, Brown D and Kauppinen E, Unambiguous atomic structural determination of single-walled carbon nanotubes by electron diffraction, Carbon, 2007; 45: 662-7.

[34] Wen J-M, Evans J W, Bartelt M, Burnett J W and Thiel P A, Coarsening mechanisms in a metal film: From cluster diffusion to vacancy ripening, Phys Rev Lett, 1996; 76: 652.

[35] Dresselhaus M S, Dresselhaus G, Jorio A, Souza Filho A G, Pimenta M A and Saito R, Single nanotube Raman spectroscopy, Acc Chem Res, 2002; 35: 1070-8.

[36] Qin L C, Ichihashi $\mathrm{T}$ and Iijima S, on the measurement of helicity of carbon nanotubes, Ultramicroscopy, 1997; 67: 181-9.

[37] Lolli G, Zhang L, Balzano L, Sakulchaicharoen N, Tan Y and Resasco D E, Tailoring (n,m) structure of single-walled carbon nanotubes by modifying reaction conditions and the nature of the support of CoMo catalysts, J Phys Chem B, 2006; 110: 2108-15.

[38] Campos D C, Belkouch J and Hazi M, Reactivity investigation on iron-titanium oxides for a moving bed chemical looping combustion implementation, Adv Chem Engineer Sci, 2013; 3: 47-56.

[39] Henry C R, Morphology of supported nanoparticles, Prog Surf Sci, 2005; 80: 92-116. 
[40] Harutyunyan A R, Chen G, Paronyan T M, Pigos E M, Kuznetsov O A, Hewaparakrama K, et al., Preferential growth of single-walled carbon nanotubes with metallic conductivity, Science, 2009; 326: 116-20.

[41] He M, Amara H, Jiang H, Hassinen J, Bichara C, Ras R H A, et al., Key roles of carbon solubility in single-walled carbon nanotube nucleation and growth, Nanoscale, 2015; 7:20284-8.

[42] Tauster S, Strong metal-support interactions, Acc Chem Res, 1987; 20: 389-94.

[43] Tatarchuk B, Chludzinski J, Sherwood R, Dumesic J and Baker R, Controlled atmosphere electron microscopy investigation of iron supported on titania, J Catal, 1981; 70: 433-9.

[44] Wang Y, Luo Z, Li B, Ho P S, Yao Z, Shi L, et al., Comparison study of catalyst nanoparticle formation and carbon nanotube growth: support effect, J Appl Phys, 2007; 101: 124310.

[45] Diarra M, Zappelli A, Amara H, Ducastelle F and Bichara C, Importance of carbon solubility and wetting properties of nickel nanoparticles for single wall nanotube growth, Phys Rev Lett, 2012; 109: 185501. 